\title{
S_Kernel: A New Symmetry Measure
}

\author{
Vito Di Gesù ${ }^{1,2}$ and Bertrand Zavidovique ${ }^{2}$ \\ 1 DMA, Università di Palermo, Italy \\ 2 IEF, University of Paris XI, ORSAY, France \\ digesu@math.unipa.it, zavido@ief.u-psud.fr
}

\begin{abstract}
Symmetry is an important feature in vision. Several detectors or transforms have been proposed. In this paper we concentrate on a measure of symmetry. Given a transform $S$, the kernel $S K$ of a pattern is defined as the maximal included symmetric sub-set of this pattern. It is easily proven that, in any direction, the optimal axis corresponds to the maximal correlation of a pattern with its flipped version. For the measure we compute a modified difference between respective surfaces of a pattern and its kernel. That founds an efficient algorithm to attention focusing on symmetric patterns.
\end{abstract}

Keywords: Symmetry transforms, symmetry measure, correlation, feature extraction.

\section{Introduction}

Symmetry is a prevalent perceptive feature for humans. For instance the human face or body is approximately symmetric that is exploited to assist in face recognition and facial feature detection. Asymmetry is instinctively interpreted for a sign of disease. Psychologists of the Gestalt school have assigned a relevant role to symmetry in attentive mechanism both in visual and auditory systems [1]. For example, psycho-physical experiments show that infants (1-2 years old) tend to fixate symmetrically around an arbitrary single distinctive stimulus (ex. corners of a triangle). From the survey by Zabrodsky [2], we stress upon some saliency of the vertical symmetry associated with a mental rotation of linear complexity with the angle. Symmetry detection skills are ranked in the order vertical, horizontal, bent and then rotational. Moreover, symmetry of parts near the axis contribute more than symmetry of further parts near edges, themselves more critical than regions in between, and half a pattern is really scanned as soon as some symmetry was conjectured. That corroborates our own findings in the machine domain.

Like most visual features as edges, regions from color or texture, or motion, symmetry appears more an impression and a clue on some phenomenon than really a quantified variable. In psychophysics, the concern for symmetry measures emerges as early as in the late fifties [3] . They should help predicting response to (plane) figures. Thus the question of binary vs. continuous concept is raised: is a pattern symmetric or asymmetric, or could it be symmetric to a certain degree? 
Zusne [4] exemplifies a research school that tends to evaluate symmetry based on moments - the third in his case - of area or perimeter of the pattern itself or of its projections. Moments can be determinate or statistical. Partly by-product of the Gelstalt theory, the informational concept of redundancy bridges both types. Single form symmetry involves redundancy, but also the shape is likely considered an item in a sample which the "best" figure is extracted from (e.g. maximum self overlapped). When symmetry is assumed single form parameter, the shape is broken into quadrants thanks to tentative axes and for instance a variance of portions in different quadrants is computed. Still in the informational vein of psychophysics studies, Yodogawa [5] departs from simple measures of the type "number of axes", "size of equivalent sub-set" etc. that usually involve frequencies (e.g. Fourier or Gabor). Redundancy is again taken for the measurable property, and the 2-D Walsh-Hadamar transform is preferred from noticing that its basic functions fall equally into 4 subsets along with the type of symmetry vertical, horizontal, $\pi$ rotational and double - . Hence the transform decomposes any single pattern into four symmetry components which an entropy function of the power spectrum is applied to, resulting in the so-called symmetropy. However, test patterns are small blocks of dots or squares, making it easy to compare with human responses but telling little about the method to process real images.

The concept of symmetry is important in machine vision too. Several surveys have been proposed, a recent quite interesting one being [6. Same as other image features, to be extracted by computers, symmetry obeys geometric or analytic mathematical models: some distance from the actual image to the model is minimized. In the present paper we rather introduce a new symmetry measure based on the concept of kernel $(S K)$ of a pattern, defined as the maximal included symmetric sub-set of this pattern 32.

Section 2 provides a review on symmetry works. In section 3 , we introduce the notion of a "kernel" that stems logically from IOT ([32]) through a classical gauge in functional analysis, and a preliminary study of the main parameter the optimal axis to spot - leads to an ultimate detection algorithm based on correlation and to a general enough symmetry measure. A series of experiments in section 4, on both binary and grey scaled pictures, show the relevance of primary results. Concluding remarks are given in Section 5.

\section{State of the Art}

The Symmetry Axial Transform (SAT) 7] can be considered one of the first approaches for the detection of symmetry starting from the borders of a digital object. SAT is a subset of the medial axis, derived from the selection of the center of maximal circles for inclusion. SAT is able to retrieve only maximal axes of symmetry, i.e. those symmetries that are already included in the medial axis. Some limitations of the SAT algorithm have been solved by the introduction of the Smoothed Local Symmetry [8]. The main idea is retrieving a global symmetry (if it exists) from the local curvature of contours, through the locus of mid-edge-point pairs. An innovating approach by Sewisy [9] makes a late avatar of that algorithmic line. 
Authors couple the Hough transform with geometric symmetry to exhibit candidate ellipse centers. All remaining feature points are grouped into sub-regions where ellipse-like patterns are actually detected. In 10 polygonal approximations of pattern contours are broken into elements (e.g. with Delaunay/Voronoi type of method) of which axial features are pieced together. In [11/12 13 the mathematical background to extract object skewed symmetries under scaled Euclidean, affine and projective images transformation is proposed. An algorithm for backprojection is given and the case of non-coplanarity is studied. The authors introduce also the concept of invariant signature for matching problems.

The use of gray level information was firstly investigated in [14, where the symmetry descriptor of a given object is based on a cross correlation operator evaluated on the gray levels. In [15], the search for symmetries is based on the evaluation of the axial moment of a body around its center of gravity. In the case of images, gray levels are considered the point masses. This descriptor has been applied at a local level to define the Discrete Symmetry Transform (DST). Object symmetries can hence be studied with axial moments of regions previously selected. In [16], local symmetry is computed in convolving with the first and second derivative of Gaussians to detect reflectional symmetry. Both a "measure" of symmetry and an axis orientation are provided at each point. Shen [17] or DuBuff [18 use complex moments associated with Fourier or Gabor transforms of images for an approximation. Although it implies axes to pass through the center of mass, and although they are not invariant to affine transforms, the phase of such (generalized) complex moments proves efficient in detecting most axes of isolated, centered and well contrasted figures.

In [19], authors introduce several descriptors from Marola's one, further extended to finite supports and varying scales for the analysis of object symmetries that are based on the Radon and the Fourier transforms. Scale dependency is claimed to detect global symmetries in an image without using any segmentation procedure. The proposed algorithm is based on a global optimization approach that is implemented by a probabilistic genetic algorithm to speedup the computation. Along the same line, Shen and al. 20] detect symmetry in seeking out the lack of it. A measure is defined that is comprised of two terms - symmetric and asymmetric energy. The latter is null for a set of pixels invariant through horizontal reflection. Hence, the method consists of minimizing the asymmetric term of the energy over an image. In [21, a multi-scale similar idea (see also [22]) is considered: a vector potential is constructed from the gradient field extracted from filtered images. Edge and symmetry lines are extracted through a topographical analysis of the vector field (i.e. curl of the vector potential). This is performed at various heights above the image plane. Symmetry axes are lines where the curl of the vector vanishes and edges are where the divergence of the potential vanishes.Yeshurun and al. 23 build on the Blum-Asada vein, but in quantifying a potential for every pixel to be center -or part of an axisof symmetry, based on pairs of edge points tentatively symmetric from their respective gradient vectors. A degree of symmetry is assigned to every pair within a given pixel neighborhood and a weighted combination of these makes the pixel 
potential, whose local maxima provide a measure depending on both intensity and shape. The technique further extends to textures [24].

Some described methods provide more symmetry descriptors that measures can be computed from, others aim at straight symmetry measures. The difference is obvious when comparing for instance Cross's and Yeshurun's. One major reason is the objective mathematical difference between a metric and a measure, the first is some integral version of the second. Another reason is whether it is required for measures in pattern recognition to mimic perceptual evaluations. An analysis of the wider concept of similarity is proposed in 25. Authors challenge distance axioms as for comparing patterns or pattern and ideal model. Tversky's psychological indexes are used to substitute distances with ordinal structures based on three properties - dominance, consistency and transitivity - that rely extensively on the "max". Hence, through set theory a connection is made with fuzzy sets to implement comparisons between segments from shapes or textures. The comparison departs mainly from others in that not only absolute segment properties as length or coarseness found it but also inter relations between neighbor segments. That was targeted and makes it interesting for any other image feature as symmetry. Tuzikof [26] designs an illustration of such concepts applied to symmetry sensed by machines. They propose to consider the co-volume of a shape $P$ and its transformed version $\tau P$, to quantify the invariance through that transform $\tau$. The co-volume is the symmetric term in the expression of the volume of the Minkowski's sum (morphological expansion) of $P$ and $\tau P$, and the measure is the co-volume normalized by the product of volumes. That makes it a symbolic correlation of $P$ and $\tau P$. So, the link is made with inertia and further with the canonical form to support proofs of invariance. The developed theory applies only to compact convex sets but it provides a perfect example of a true symmetry measure that takes advantage of not obeying the triangle inequality.

Zabrodsky 27 tackles explicitly symmetry detection as a continuous feature. She selects equidistant points along contours or equiangular edge points around the pattern center-of- mass (centroid). From these $n$ points the nearest $C_{n}-$ symmetric pattern is built in rotating the average of the counter rotation version of the points by $2 i \pi / n,[0 \leq i \leq n-1]$. The method extends easily in 2.5 -D by facets, and to grey level objects in treating inner level lines separately the same way. The measure considered is the point to point $L_{2}$-distance from the pattern to its nearest symmetric version. An iterative procedure of the gradient descent type can improve the center of symmetry by maximizing the measure, in case of a predicted pattern truncation. In the many applications they completed in Chemistry, corners seem to be favored as most as possible for the point basis. Yet the method remains line based if not strictly edges. It depends heavily on the centroid and on the choice of points, hence of the order, for which optimality appears hard to provide. About the order, an interesting comment by Kanatani [28] reminds that symmetries make an hierarchy. Then for a given set of $n$ points, information criteria exist to ground a maximization of the robustness to perturbations. An optimal order of symmetry can thus be derived. That is also an answer to those who claim that moments are the only order 
free technique. Yet an other answer to the same is that the hierarchy justifies to privilege vertical then horizontal symmetry as nature does. Apart from the relativity of symmetry direction, the review of preceding works points out that: 1) searching for invariance in comparing a pattern with its transformed version, prevents from imposing the centroid for the a priori focus of interest; 2) introducing true measures helps building more abstract versions of distances, more suitable for approximate comparison of objects; 3) sets which measures apply on may be "sets of pixels or vectors" making the shapes under consideration or "sets of patterns comparable through some class of transforms": in either case "set operations" , as Minkowski's ones, are worth considered. They do not limit to contours and bridge logic with geometry.

Before to show in the next sections why and how we put these ingredients together, we conclude this bibliography by recalling three more works that fit very well the algorithmic line above and are the closest to ours. It makes clear the main contributions of this paper respective to previous work. In [29] the authors had this intuitive idea of correlating the image with its transformed version to by-pass the centroid. But they do that on the inner product of (gaussian) gradients, hence on edges. R. Owens [30] searches explicitly for a measure of symmetry to indicate approximate bilateral symmetry of an extracted object. But she defines tentative symmetries from the principal axes of inertia, whence the centroid again, before to compute the sum of absolute differences of grey levels in symmetric pairs over the object, normalized by their maximum. When the symmetric point falls out of the object the difference is set to this latter maximum value. Note that, although it is not mentioned, such a measure amounts to a slightly modified $\mathrm{L}_{1}$-difference between the object and a maximal-for-inclusion symmetric version of it in the given direction. Kazhdan et al. 31] target a true visualization of symmetry over every point of the pattern. They use explicitly the same idea of a difference $\left(\mathrm{L}_{2}\right.$ in their case) between the image and its closest symmetric version, proven to be the average of the picture and its transform. But they need a measure that integrates all reflective invariance about a bundle of straight lines (or planes in 3-D). It is robust to noise and suitable for object matching, yet a center is necessary to this symmetry representation. The representation plots for each and every direction the measure of symmetry about the plane normal to that direction passing through the center of mass. Note that its local maxima point out the potential pattern symmetries.

\section{The New Symmetry Measure}

In previous papers 32 we defined the IOT that is a map product of iterated morphological erosion and symmetry detection.

Definition 1. The Symmetry Transform, $S$, on a continuous object $X \subset R^{2}$ is given by:

$$
S_{\alpha}(\mathrm{X})=\int_{X} m(x) \times \rho^{2}(x, r(\alpha)) d x \quad \text { for } \quad \alpha \in[0, \pi[
$$


where, $r(\alpha)$ is the straight line with slope $\alpha$ passing through the center of gravity of the object $X, m(x)$ is the mass of the object in $x \in X$, and $\rho$ is a distance function of $x$ from the straight line.

Definition 2. The Iterated Object Transform, IOT, is given by:

$$
\operatorname{IOT}_{\alpha, 1}(X)=S_{\alpha}(X), \operatorname{IOT}_{\alpha, n}(X)=S_{\alpha}\left[(\mathrm{E})^{n-1}(X)\right] \quad \text { for } n>1
$$

$(\mathrm{E})^{n}$ stands for the morphological erosion by the unit sphere (or any other suitable structuring element would any suitable a priori information be available), iterated $n$ times. The number of iterations depends on the size of the input image and on the distribution of the gray levels. The $S$ transform is thus computed on progressively shrunk versions of the binary input image or on steadily intensity reduced versions of the gray level input image, until some predefined decrease or a minimum of intensity is reached.

\subsection{Definition of the Kernel}

Following commonly used gauges in functional analysis, a possible object to support a symmetry measure could be maximal included symmetric pattern resp. minimal including symmetric pattern : extremal then subjects to the measure.

Definition 1. The $S$-kernel of the pattern $P-S K(P)$ - is the maximal for inclusion symmetric (pattern) subset of $P$ (see Fig. 1).

Remark 1: the center of mass likely varies from the kernel to the pattern.

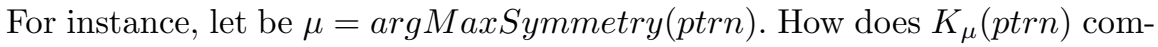
pare with $K(p t r n)$ ? How do their respective Symmetry relate? In most cases $K_{\mu}(p t r n)$ should be a good enough gauge of $K(p t r n)$, or the difference between them will be most indicative.

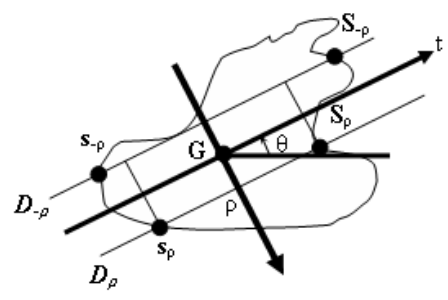

(a)

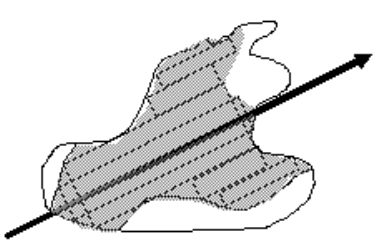

(b)

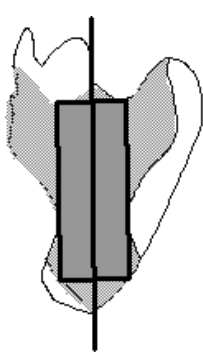

(c)

Fig. 1. (a) Sketch of the kernel detection algorithm; (b) the kernel of the pattern in (a); (c) expanding the IOTK of the pattern in (a) into the kernel 


\subsection{Formal Setting and an Algorithm}

Considering operators to be used in the end, correlation, our conjecture here is of an attention focusing process based on symmetry. Introducing the latter notion of interest implies that all non interesting regions around be faded to zero, and that the pattern scan be started for instance at the very left of it. Hence, the pattern $f(x)$ gets a bounded support and the origin of the $\mathrm{x}$-axis can be where the interest starts.

Let be (Fig 2): $S_{f}^{x}(t)=\frac{f(x+t)+f(x-t)}{2}$ the symmetric version of $f$ with respect to $x$. For the $L_{2}$-norm, the best axis $x^{*}$ corresponds to:

$$
S_{x *}(f)=\min _{x} \int_{a}^{b}[f(x+t)-f(x-t)]^{2} d t
$$

It is easily proven that $S_{x^{*}}(f)$ corresponds to

$$
(f \otimes f) \text { maximal } \quad \text { or equivalently to } \int_{\sup (0,2 x-b)}^{i n f(2 x, b)} f(2 x-u) \times f^{\prime}(u) d u=0
$$

One gets yet another algorithm: find the maximum correlation of the picture in a given direction (i.e. over all translations in this direction) with its mirror symmetric in that direction (i.e. scanned right to left). Considering existing efficient algorithms for image geometric transforms (eg. cordic), rotations to span directions can then be performed on the image before scans and correlation: approximations need to be checked for the error introduced remain acceptable (comparable to the one from sampled angles and discrete straight lines).

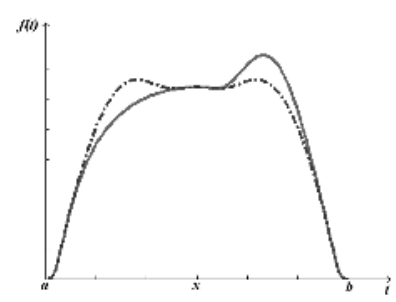

Fig. 2. Searching for the symmetry axis of a function $y=f(t)$ (plain line): its symmetric version around $x, S_{f}^{x}(t)$ (dotted line)

Remark 2: Note that if the image is tiled adapted to the predicted size of potential symmetric objects, one can derive an efficient focusing process.

\subsection{Symmetry Measure}

Following the preliminary simplifying assumption above, while the kernel is exhibited the picture is considered to be binarized or at least the pattern was cropped if it was not before. So, in order to test the proposed algorithm we compute a measure of symmetry classically defined as: $\lambda_{1}=1-\frac{\operatorname{Area}(D)}{\operatorname{Area}(A)}$, with $A$, the pattern or a binding sub-picture, $B$, its kernel, and $\operatorname{Area}(D)=\operatorname{Area}(A-B)$. Provided suitable binarization or windowing, it remains a robust first approximation where $\lambda_{1}=1$ if $\operatorname{Area}(B)=\operatorname{Area}(A)$. 


\section{Experimental Results and Further Comments}

In this section we show some results of the application of the S-kernel algorithm $(S K A)$ to attention focusing.

We tested the possibility of using kernel based operators to detect points of interest in complex images. Examples of such images are shown in Fig.s 3 a,b; they represent a famous painting by Tintoretto and a group photo under natural illuminating conditions. In both images the goal was to detect the directions of the most symmetric objects of a given size. For example in the group photo the direction of people faces (see Fig. [3r,d).

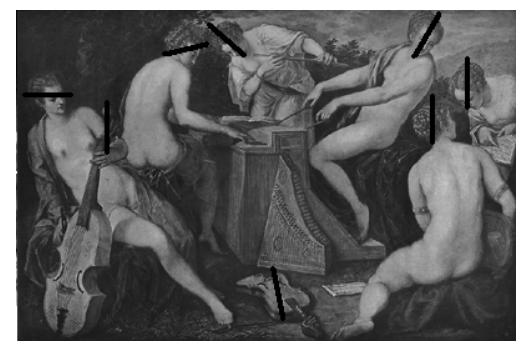

(a)

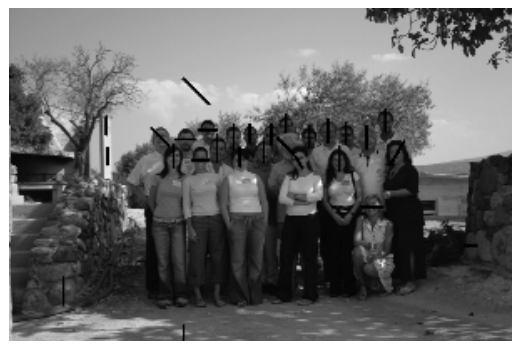

(b)

Fig. 3. Point of attention derived by the kernel transformation based on the correlation for images: (a) Group of women (Tintoretto 1545-1588); (b) group photo

The implemented algorithm is not general: the size of the target is first estimated. This is not a big constraint in many applications, among which is face detection. The procedure consists in raster scanning the input image with a window, size of which is set on the basis of the human face dimensions scaled to the input frame. Inside each window kernel-based operators are computed. The algorithm returns all windows for which the value of $\lambda(\rho)$ is greater than a given threshold $\phi \in[0,1]$. Here, the threshold was set to the mean value of $\lambda(\rho)$ in all experiments. A great value of $\lambda(\rho)$ in a given direction indicates a bilateral symmetry typical of face like objects. Fig.s 3r,d show the results from $S K A$. Not all objects with high bilateral symmetry are faces. Nevertheless the method was able to extract all face positions, introducing an error of $17 \%$ in the evaluation of the face direction. And over all experiments the percentage of not faces was $21 \%$.

\section{Concluding Remarks}

This paper describes a new measure of axial symmetry derived from a new object feature named the "symmetry-kernel". The symmetry kernel of an object is its maximal subpart symmetric respective to a given direction. A new algorithm 
is derived from, based on the computation of the cross-correlation of an object with its flipped version. It is fast and not sensitive to numerical factors because computations are inner products. The algorithmw as tested on both synthetic and real data. Experiments show the ability of the symmetry-kernel to detect the main directionality of an object. It has been also implemented as a local operator to detect the presence of objects in a scene and their direction. The evaluation of the distance between an object and its kernel is a crucial point and needs further investigation.

\section{References}

1. W.Khöler and H.Wallach, "Figural after-effects: an investigation of visual processes", Proc. Amer. phil. Soc., Vol.88, pp.269-357, 1944.

2. H. Zabrodsky, "Symmetry - A review", Technical Report 90-16, CS Dep. The Hebrew University of Jerusalem, 1990.

3. R.M. Boyton, C.L. Elworth, J. Onley, C.L. Klingberg, "Form discrimination as predicted by overlap and area", RADC-TR-60-158, 1960.

4. L.Zusne, "Measures of symmetry", Perception and Psychophysics,Vol.9, N.3B, 1971.

5. E. Yodogawa, "Symmetropy, an entropy-like measure of visual symmetry", Perception and Psychophysics, Vol. 32, N.3, 1983.

6. David O'Mara, "Automated facial metrology, chapter 4: Symmetry detection and measurement" PhD thesis, Feb. 2002.

7. H.Blum and R.N.Nagel, "Shape description using weighted symmetric axis features", Pattern recognition, Vol.10, pp.167-180, 1978.

8. M.Brady, H.Asada, "Smoothed Local Symmetries and their implementation", The International Journal of Robotics Research, Vol.3, No.3, pp.36-61, 1984.

9. A. Sewisy, F. Lebert, "Detection of ellipses by finding lines of symmetry inthe images via an Hough transform applied to staright lines", Image and Vision Computing, Vol.19, N.12, pp. 857-866, 2001.

10. S. Fukushima, "Division-based analysis of symmetry and its applications", IEEE PAMI, Vol.19, N.2, 1997.

11. D.P.Mukhergee, A.Zisserman, M.Brady, "Shape form symmetry: detecting and exploiting symmetry in affine images", Philosofical Transaction of Royal Society of London Academy, Vol.351, pp.77-101, 1995.

12. T.J.Chan, R.Cipolla, "Symmetry detection through local skewed symmetries", Image and Vision Computing, Vol.13, N.5, pp.439-455, 1995.

13. J.Sato, R.Cipolla, "Affine integral invariants for extracting symmetry axes", Image and Vision Computing, Vol.15, N.5, pp.627-635, 1997.

14. G.Marola, "On the detection of the axes of symmetry of symmetric and almost symmetric planar images", IEEE Trans.of PAMI, Vol.11, pp.104-108, 1989.

15. V.Di Gesù, C.Valenti, "Symmetry operators in computer vision", in Vistas in Astronomy, Pergamon, Vol.40, N.4, pp.461-468, 1996.

16. R. Manmatha, H. Sawhney, "Finding symmetry in Intensity Images", Technical Report, 1997.

17. D. Shen, H. Ip, K.T. Cheung, E.K. Teoh, "Symmetry detection by Generalized complex moments: a close-form solution", IEEE PAMI, Vol. 21, N.5, 1999. 
18. J. Bigun, J.M.H. DuBuf, "N-folded symmetries by complex moments in Gabor space and their application to unsupervized texture segmentation" IEEE PAMI, Vol. 16, N.1, 1994.

19. N.Kiryati, Y.Gofman, "Detecting symmetry in grey level images (the global optimization approach)", preprint, 1997.

20. D. Shen, H. Ip, E.K. Teoh, "An energy of assymmetry for accurate detection of global reflexion axes, Image Vision and Computing, Vol.19, pp.283-297, 2001.

21. A. D. J. Cross and E. R. Hancock, "Scale space vector fields for symmetry detection", Image and Vision Computing, Volume 17, N.5-6, pp. 337-345, 1999.

22. V.Di Gesù, C.Valenti, "Detection of regions of interest via the Pyramid Discrete Symmetry Transform", in Advances in Computer Vision (Solina, Kropatsch, Klette and Bajcsy editors), Springer-Verlag, 1997.

23. D.Reisfeld, H.Wolfson, Y.Yeshurun, "Detection of interest points using symmetry", 3rd IEEE ICCV Osaka, 1990.

24. Y. Bonneh, D.Reisfeld, Y.Yeshurun, "Texture discrimination by local generalized symmetry", 4th IEEE ICCV Berlin, 1993.

25. S. Santini, R. Jain. "Similarity measures" , IEEE PAMI Vol.21, N.9, 1999.

26. H.J.A.M. Heijmans, A. Tuzikof, "Similarity and Symmetry measuresfro convex shapes using Minkowski addition", IEEE PAMI , Vol.20, N.9, pp.980-993, 1998.

27. H. Zabrodsky, S. Peleg, D. Avnir, "Symmetry as a continuous feature", IEEE PAMI, Vol.17, N.12, 1995.

28. K. Kanatani, "Comments on Symmetry as a continuous feature", IEEE PAMI, Vol.19, N.3, 1997.

29. T. Masuda, K. Yamamoto, H. Yamada, "Detection of partial symmetyr using correlation with rotated-reflected images", Pattern Recognition, Vol.26, N.8, 1993.

30. D. O'Maraa, R. Owens, "Measuring bilateral symmetry in digital images". IEEE TENCON, Digital signal processing aplications, 1996.

31. M. Kazhdan, B. Chazelle, D. Dobkin, A. Finkelstein, T. Funkhouser, "A reflective symmetry descriptor", $7^{\text {th }}$ ECCV, pp. 642-656, 2002.

32. V. DiGesu and B. Zavidovique, "A note on the Iterative Object Symmetry Transform", Pattern Recognition Letters, Pattern Recognition Letters, Vol.25, pp.15331545, 2004. 